\title{
Solving the narrative paradox in VEs - lessons from RPGs
}

\author{
Sandy Louchart, Ruth Aylett \\ Centre for Virtual Environments, University of Salford M5 4WT \\ \{S.Louchart, R.S.Aylett\}@ Salford.ac.uk
}

\begin{abstract}
Narrative within Virtual Environments (VEs) is a compromise between pre-authored narrative structures and user freedom in terms of interaction and physical movement. We present results of a recent investigation on the narrative structures and mechanisms of Role Playing Games (RPGs), and assess the potential of non-conventional narrative forms for computer implementation. We address the potential benefits for the research currently undertaken by the AI community in terms of storytelling and interactive storytelling.
\end{abstract}

\section{Introduction}

The concept of a 'narrative paradox' in virtual environments (VEs) is now well established [1]. This revolves around the conflict between pre-authored narrative structures - especially plot - and the freedom a VE offers a user in physical movement and interaction, integral to a feeling of physical presence and immersion. This paper extends work already carried out overviewing the concept of emergent narrative [2], assessing the relevance of current narrative theory [3], and examining the possible basis for a narrative theory particular to the medium of VEs which could underpin narrative applications [4]. Since a large part of the narrative action in a VE is related to the actions of synthetic characters, the narrative framework adopted is of great significance for the architecture and functionality of such characters, from the internal action selection mechanism to external expressive behaviour. We argue that the defining characteristic of VEs is not their visual realism, which might make the narrative approach of film or television the most relevant, but their interactivity. For this reason, rather than trying to adapt classical narrative theories $[5,6,7,8]$ we are investigating the structures and mechanisms of less-studied but potentially relevant interactive media. Flowing from interactivity is the participative character of narrative in a VE - thus we focus on narrative as an experience for the user rather than as a spectacle. Our Emergent Narrative [2] concept focuses and tries to capitalise on the entertainment values of discovery, interaction and immersion and is being developed in the belief that the audience, in this case the users, can through their interactions with Virtual agents or Virtual actors, participate in the emergence of a narrative that would be both coherent and satisfying as an experience at an individual level. Such a narrative may not conform to the ideology of narrative as a spectacle and might not be dramatically suitable for simple screenings. 


\section{Role Playing Games}

The motivation for this study of Role Playing Games [RPGs] was to derive elements of a possible solution to the narrative paradox and an approach to reconciling plot structure, plot line, and the level of freedom offered to the user in terms of space, time and interaction. RPGs cannot easily be characterised in terms of standard narrative theories, presenting a different approach to narrative. Their interactive character-based approach differs both from the classical Aristotelian [5] theory and the analytical models proposed by the French Structuralists [6, 7 8].

Our investigation aimed at identifying narrative patterns, elements or factors influencing the creation, development and unfolding of dramatic narratives and stories. However, it is not possible to base such a study on existing literature since there appears to be very little other than specific campaign role-playing setting books. A protocol-based approach would involve observing and documenting actual games, but this is practically demanding and potentially difficult to generalise. The selected approach to this study was therefore knowledge acquisition through elicitation from an expert. The results displayed in this paper are based on sessions conducted with $\mathrm{Dr}$ Ian McBriar, an experienced RPG Games Master and the 2001 World Champion of Ultra Modern World Team Championships, who presented at the NILE02 [9] on the subject of Role Playing Games.

Knowledge acquisition is known to be a difficult and time-consuming activity. Empiricom Ltd (http://www.empiricom.co.uk) [10] made their $\mathrm{KAT}^{\mathrm{TM}}$ Builder software [11], as well as essential training, available to us for this study via utilisation of the $\mathrm{KAT}^{\mathrm{TM}}$ Technique and $\mathrm{KAT}^{\mathrm{TM}}$ Builder software. The process known as "Knowledge Elicitation" is that of actually getting tacit knowledge out of a human expert and putting that knowledge into a form which is computable, that is, a format suitable for use by a computer system. Empiricom's Knowledge Acquisition Technique (KAT ${ }^{\mathrm{TM}}$ ) applies a highly rigorous logical formalisation of the philosopher Karl Popper's "falsificationism" [12]. Essentially, it states that the most efficient way to solve a problem is not to try to find all of the conditions that must be true for a hypothesis to hold, but rather seek out only the evidence which would disprove a hypothesis.

\section{Results of the study}

The creation of a campaign is a collaborative process where the characters, as well as the worlds and environments in which the campaign is set, are developed in common accord between the Game-Master and the players. Character definitions include details such as histories, activities, work, physical characteristics or eating habits. Environments and worlds are defined with the same level of detail. This laborious but highly participative creation process allows the Games Master to prepare the campaign episodes with a good understanding and knowledge of the different characters and worlds involved. This favours the delivery of a highly flexible 
narrative structure, potentially challenging all the different protagonists of the party while addressing the importance of roles in narrative structures.

During the course of the campaign, RPG players are confronted with a certain number of encounters [Table 1], distributed in time and space by the Game-Master as a source of challenging and interesting activities for the party. Their presence in any game is, however, dependent on the genres and themes of the campaign and its specific settings.

\begin{tabular}{|l|l|}
\hline \multicolumn{1}{|c|}{ Name } & \multicolumn{1}{c|}{ Content } \\
\hline Descriptive & Game-Master description of states rules; functions, scene. \\
\hline Social & NPC communicates information in a social context \\
\hline Information-gathering & $\begin{array}{l}\text { NPC assesses state of player's knowledge for gaps; } \\
\text { provides information or clue }\end{array}$ \\
\hline Problem-solving & NPC confronts player with puzzle or problem \\
\hline Combat & NPCs for action encounters - battles, fighting \\
\hline
\end{tabular}

\section{Table 1: RPG encounter types}

The Game-Master expects that the encounters specifically created for a session, will trigger actions, reactions, discussions or decisions from the party in such way that an anticipated plot will unfold. This plot however has a hypothetical aspect since what actually happens is the direct result of the party's generated reactions to the different encounters. They can be used by the Games Master to shape and pace the dramatic unfolding of the narrative as well as presenting the main source of entertainment to the players, and embodying key events in the construction of the plot. Their smooth orchestration by the Game-Master is critical to the creation, development and unfolding of an RPG campaign.

We have already argued [2] that a hierarchical view of narrative allows us to separate out issues of control into different levels of abstraction and had suggested different levels. Control within RPGs can be viewed as dynamic negotiation across these levels. The Game-Master exercises control at a high level over narrative unfolding, plot, pace and structure of the story. Since the a priori plot line for a campaign is only hypothetical, the Game-Master needs specific tools - in the form of the encounters just described - to gain some control over the overall campaign. The players, through their actions, decisions, strategies, the skills of their character and their own personal capabilities to act in role, produce the content of all levels from abstract action downwards. The players, though they do not carry any narrative responsibility, exercise control at a character and individual level, whereas the GameMaster is in charge of issues of greater narrative importance.

\section{Ensuring the progress of the story}

This is achieved in the main through a wise use of the different encounters available to the Game-Master. Framing a hypothetical scenario, a Game-Master writes and develops each episode's encounters as the campaign unfolds, from session to session and may dynamically introduce specific actions if the delivery of an interesting story or control over the overall narrative seems threatened. Interventions are generally caused either by players taking longer than expected in dealing with encounters or by the story branching in an unexpected manner. Branching may occur 
when the party incorrectly determines their role and what is expected from them, pursues future plot events omitting essential encounters or attempts to reinvent themselves The need for interventions illustrates the plot's provisional nature and stresses on the Game-Master's preparation and flexibility. The Game-Master first assesses the potential value of new resulting sub-plots for the party, decides whether or not this allows the campaign to continue, and if not takes appropriate actions

\section{Ensuring the satisfaction of the party}

In entertainment of nearly any form, there is always at some stage the idea of the targeted audience in the minds of theatre directors, novelists or film directors/ screenplay writers. The same could be said of the Game-Master even though the common values of theatre, novel and cinema do not obviously apply to a participative narrative form such as RPG. What matters for a spectator might not match the priorities of a character in a participative environment. Although RPG players have a good idea of the overall story in which they are involved, they are more concerned by the development of their characters and their focus is situated at a fairly low level within the overall story, the individual level. It is therefore important that the GameMaster monitors players' behaviours both in and out of character, and decides corrective measures if it appears that certain players are not enjoying the game.

\section{Conclusions}

This study of RPGs was carried out specifically to see how far, as an interactive medium, it provided solutions to the narrative paradox [13] in VEs. We have shown that this system combines a certain freedom at the individual level of character role play with a relatively satisfying level of control over the overall story for the author, the Game-Master. It seems to offer a good compromise between the freedom exercised and experienced by the user/player and the narrative control necessary for the development and unfolding of interesting stories, though not relying as much on characters as other narrative forms such as Interactive Theatre [14]. Relevant work here is that of Mateas and Stern in their Façade system [15] though their concept of beats operates at a far lower level of abstraction than the interface between GameMaster and players in RPGs. Like every structure or mechanism the RPG approach has weaknesses and strengths. A VE implementation would require not only NPCs of typically greater richness than in current computer-based RPGs but also persistent synthetic players to form the overall party. Simulation of individual and low-level (character level) narrative control within the model seems feasible to some extent, thus incorporating a continuing interaction memory. Some of the high-level decision control (Game-Master/referee level) also seems feasible, though the interventions dealing with narrative or individual difficulties clearly draw on human judgement and creativity beyond the current state-of-the-art in synthetic characters. Although the signs of narrative impasse can be relatively easily categorised, it would be much more difficult to build a computational system able to assess and act on users' satisfaction levels. We believe that, using encounters, the role played by the Game-Master could be considered somewhat between that of a theatre or cinema director whose plot 
events are staged and that of an IMPROV artist who has to deal with the situation live and in real time in the majority of cases, that is, a real-time director. The dynamic authoring carried out by the Games-Master within an episode corresponds to a continuous planning-replanning system [16], while the design of encounters for the next episode requires hierarchical expansion from a high-level campaign. Although RPGs work with a hypothetical plot which is dynamically modified, the mechanisms supporting this dynamic modification seem to rely too much on out-of-character and out-of-play direct interactions. Other interactive narrative forms remain to be studied, with Interactive Theatre [14] of particular interest for a further assessment of the relationships between plot and characterisation. Interactive theatre seems to have developed precisely in order to accommodate to the constraints imposed by interactivity and spectator freedom, making it very relevant to a concept of Emergent Narrative

\section{References}

[1] Aylett, R (2000) Emergent Narrative, Social Immersion and "Storification"; Proceedings Narrative and Learning Environments Conference NILE00 Edinburgh, Scotland.

[2] Aylett, R. (1999) Narrative in virtual environments - towards emergent narrative. 1999; AAAI Symposium on Narrative Intelligence pp 83-86

[3] Louchart S, Aylett R (2002) Narrative theories and emergent interactive narrative; Proceedings Narrative and Learning Environments Conference NILE02 Edinburgh, Scotland pp 1-8

[4] Aylett R, Louchart S. (2003) Towards a narrative theory of VR. Virtual Reality Journal, special issue on storytelling. To appear 2003.

[5] Aristotle. (1987) The Poetics of Aristotle, translation and commentary by Stephen Halliwell 330 B.C. Duckworth, 1987

[6] Barthes R. (1981) Communication 8, Introduction à l'analyse structurale des récits. 1966. Editions du Seuil.

[7] Todorov T (1970) Grammaire du Decameron. 1970. The Hague. 1970.

[8] Propp V (1928) Morphology of the Folktale. 1928. University of Texas Press, 1998

[9] McBriar. I, (2002) Invited talk: Building Believable Worlds: A gamesmasters perspective. NILE02. 2002

[10] Empiricom Limited. http://www.empiricom.co.uk

[11] Butler Group. KATTMAcquire, KATTMBuild, KATTMConsult - v1.0 (Research Paper Dec 2001)

[12] Popper K.(1959) The logic of scientific enquiry. Hutchinson, London UK. 1959.

[13] Theune M, Faas S, Nijholt A, Heylen (2003) The Virtual Storyteller: Story Creation by Intelligent Agents; Proceedings Technologies for Interactive Digital Storytelling and Entertainment TIDSE 2003 pp 204-215.

[14] Izzo Gary (1997) The art of Play, the new genre of interactive theatre, Heinemann Portsmouth. 1997

[15] Mateas M, Stern A. (2003) Integrating Plot, Character and Natural LanguageProcessing in the Interactive Drama Façade; Proceedings Technologies for Interactive Digital Storytelling and Entertainment TIDSE 2003 pp 139-151.

[16] Myers KL. (1999) CPEF: A Continuous Planning and Execution Framework. AI Magazine, vol 20 no 4, pp 63-69. 